\title{
Smoking status and survival: impact on mortality of continuing to smoke one year after the angiographic diagnosis of coronary artery disease, a prospective cohort study
}

\author{
Fadi Hammal ${ }^{1}$, Justin A Ezekowitz ${ }^{2,6}$, Colleen M Norris ${ }^{2,3,4,5}$, T Cameron Wild $^{4}$, Barry A Finegan ${ }^{1 *}$, for the
} APPROACH Investigators

\begin{abstract}
Background: Smoking is an undertreated risk factor for coronary artery disease (CAD) and is associated with adverse outcomes after myocardial infarction. Aims of our study were to determine if management of CAD by medical therapy (MT) alone or with coronary artery bypass grafting (CABG) or percutaneous coronary intervention $(\mathrm{PCl})$ influence smoking status at one year following angiography and if a change in smoking status at one year influences long term survival.

Methods: Prospective cohort study using the APPROACH registry. Two cohorts were examined: (1) 11,334 patients who returned a one year follow-up questionnaire; (2) 4,246 patients propensity-matched based on their post-angiography treatment - MT or revascularization (RV). Multivariate modeling and survival analysis were used.

Results: In the propensity-matched cohort, quit rates at one year were greater among CABG patients (68\%) than $\mathrm{PCI}(37 \%)$ or MT patients (47\%). Smokers in the RV group, who self-reported quitting at one year, had a significantly reduced mortality compared to those who continued to smoke.
\end{abstract}

Conclusions: CABG patients were more likely to quit smoking than those treated with MT alone or PCl. Quitting smoking was associated with improved long-term survival; smoking remains a key risk factor for mortality in patients with CAD. These data underscore the importance of nicotine addiction management in patients with CAD and the need to emphasize cessation particularly in those patients undergoing MT or PCl.

Keywords: Smoking cessation, Coronary artery disease, Coronary artery bypass grafting, Percutaneous coronary intervention

\section{Background}

Cigarette smoking is associated with the development of coronary artery disease (CAD) [1] and influences short and long term outcomes of patients who smoke after diagnosis [2-4]. Smoking cessation substantially reduces the risk of total coronary heart disease mortality [5,6], and can reduce the need for revascularization procedures [7]. The single most common CAD risk factor among patients hospitalized for initial myocardial infarction is hypertension

\footnotetext{
* Correspondence: bfinegan@ualberta.ca

'Department of Anesthesiology \& Pain Medicine, University of Alberta, 8-120

Clinical Sciences Building, Edmonton, Alberta T6G2G3, Canada

Full list of author information is available at the end of the article
}

(44\%), followed by smoking (23\%) [8]. Globally, smoking is still considered to be responsible for up to one third of the mortality associated with cardiovascular disease [9]. Despite the availability of smoking cessation therapies and the publication of excellent guidelines outlining the measures required to enhance the quit rates of smokers with CAD [10], there continues to be poor uptake by clinicians and patients of the behavioural and pharmacological measures available to assist patients with established CAD to quit smoking [11].

The objectives of the current study were to assess 1) if different management strategies: medical therapy alone (MT), MT and coronary artery bypass grafting (CABG) 
or MT and percutaneous coronary intervention (PCI) influence quit rates at one year following diagnostic angiography, and 2) if quitting smoking at one year influences long term survival independent of the treatment strategy.

\section{Methods \\ Design and setting}

The Alberta Provincial Project for Outcomes Assessment in Coronary Heart disease (APPROACH) registry captures data on all patients who undergo coronary angiography in Alberta, Canada. This study reports on data collected between January 2003 and March 2010. Database and data collection methods have been previously described in detail [12]. In brief, data collected includes socio-demographic characteristics, smoking status, clinical co-morbidities, prior cardiac events, indication for angiography (which includes detail regarding a clinical event if present), ejection fraction, coronary anatomy and all treatments received (MT, CABG, PCI) following angiography. Clinical indications for angiography included acute/recent $\mathrm{MI}$, stable angina, unstable angina, and other. Data in APPROACH are linked quarterly with the Service Alberta Ministry-Vital Statistics to capture mortality and date of death (99.9\% follow up).

All patients in APPROACH who consented to followup during the study period were sent a questionnaire one year after their index angiography. Two questions related to tobacco: Do you currently smoke cigarettes? and If you quit, how long ago did you quit?

The APPROACH study and sub-studies are approved by the ethical review boards at the University of Alberta and the University of Calgary.

\section{Statistical analysis}

Pearson Chi-Squared test for categorical variables and t-test for continuous variables were used to compare baseline clinical characteristics across strata. Patients were initially stratified based on their smoking status at time of angiography into current smokers and non-smokers. Patients were subsequently stratified into four categories based on their smoking status at angiography and one year: 1) patients who reported being smokers at baseline and at follow-up, 2) patients who reported being smokers at baseline and non-smokers at follow-up, 3) patients who reported being non-smokers at baseline and at follow-up, and 4) patients who reported being non-smokers at baseline and smokers at follow-up. The latter constitute a small number of patients $(n=64)$, and were excluded from further analysis. A Pearson Chi-Squared test was used to assess the association between the treatment that the patient received after angiography and smoking status at one year follow-up.

Two cohorts were examined: Cohort $A$, the entire group who returned an analysable questionnaire at one year, and, Cohort $B$, a group from Cohort $A$, matched based on the propensity to undergo revascularization. The propensity score was calculated as the probability of undergoing revascularization (PCI or CABG) on the observed baseline (measured at recruitment) characteristics. This technique allows for a high number of confounding variables and has been used to create a stratum of subjects who can be matched on the propensity score whereby exposure is not confounded with measured baseline covariates. The propensity score was calculated using logistic regression. The following variables were included in the model: age, sex, smoking status, pulmonary disease, cerebrovascular disease, renal disease, heart failure, diabetes, dialysis, hypertension, hyperlipidemia, liver/GI disease, malignancy, peripheral vascular disease, prior MI, prior PCI, prior CABG, prior lytic therapy, the indication for angiography including MI, stable angina, unstable angina or other, the coronary anatomy and the ejection fraction. Greedy matching techniques were applied to match patients who were revascularized to patients who were treated with medical management by matching the participants with the nearest propensity score, i.e. within 3 decimal places of the propensity score for each case. Overlap of propensity scores between revascularized and medically managed patients were evaluated using histograms, Chi-Squared values and probability values. Differences in baseline factors between groups were calculated before and after propensity adjustment to assess balance.

Survival analysis was conducted; survival tables and log rank tests were used to determine if there was a statistically significant survival difference between revascularized and medically managed patients.

Sensitivity analysis was conducted in order to account for the effect of disease progress and medical management changes over time by splitting the follow up period into two 45 month periods.

All analyses were conducted using SPSS version 19.0 (IBM SPSS, Armonk, NY).

\section{Results}

In all 29,230 patients were invited to complete the APPROACH survey at one year after their procedure. The response rate to the survey was $57.4 \%$ with $38.7 \%$ returning a fully completed questionnaire that was suitable for analysis. Surveys were not sent to the families of subjects who had died within one year $(n=1968 ; 6.7 \%$ of total patient population), $33 \%$ of those who died were smokers at time of angiography. Data on the latter group consisted of demographic and other information collected at the time of angiography and mortality data obtained from Service Alberta Ministry - Vital Statistics.

The smoking status of at baseline and at one year for both Cohorts is shown in (Figures 1 and 2). In Cohort $A$, CABG patients were more likely to report quitting at 


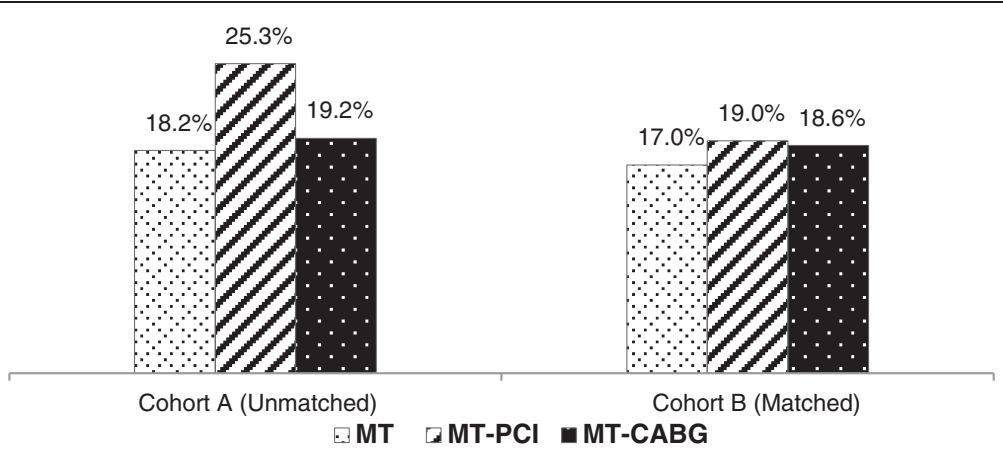

Figure 1 Smoking status at the time of angiography. MT: Medial Treatment, PCl: Percutaneous Coronary Intervention, CABG: Coronary Artery Bypass Grafting.

follow up. In Cohort $B$, groups were well matched with respect to smoking status at time of angiography and as in Cohort $A$, CABG patients were more likely to quit.

In Cohort $A$, patients who smoked were significantly younger at time of index angiography, were more likely to present with acute coronary syndrome, to had a prior MI and to have required an emergency or urgent in-hospital angiography (Table 1). These differences remained significant when patients were stratified according to postangiography treatment strategy (Table 2). Additionally, characteristics of smokers who quit compared with those who did not quit in Cohort $A$ are shown in Table 3.

The baseline characteristics of Cohort $B$ were remarkably well matched (Table 4). There were no significant differences between initial treatment strategies and subsequent cross-over to another treatment strategy - $86 \%$ and $87 \%$ of patients in MT and RV groups did not undergo any other treatment over the follow-up period (Table 5). Self-reported smoking cessation rates at one year following angiography were significantly higher among CABG patients $(68 \%)$ than PCI $(37 \%, \mathrm{p}<0.001)$ or MT patients $(47 \%, \mathrm{p}<0.001)$ (Figure 2, Table 6).

\section{Survival analysis}

Patients were followed for a mean of 42.2 months (SD 24.9 months). Non-smokers had a significantly higher long term survival rate compared with smokers in the RV group (93\% vs. 89\%, p <0.05), but not in those in MT ( $89 \%$ vs. $88 \%, \mathrm{p}=0.8)$ (Table 7). Smokers at baseline who had quit at one year had greater long term survival rate compared with those who continued to smoke after revascularization ( $95 \%$ vs. $89 \%, \mathrm{p}<0.05)$, a trend towards reduced mortality was also observed in patients in the MT group (93\% vs. $88 \%, \mathrm{p}=0.2$ ) (Table 7 ).

Our sensitivity analysis showed no alteration in the smoking-related survival pattern, however, survival improved for all patients regardless of the index treatment strategy in the second follow-up period (MT: $85.7 \%$ vs. 93.1\%; CABG: $84.5 \%$ vs. $91.2 \%$; and PCI: 91.4 vs. $96.2 \%$, first vs. second period, respectively).

\section{Discussion}

The overwhelming impact of tobacco smoking on the time course of the appearance of CAD is illustrated in our study, confirming the findings of the National Registry

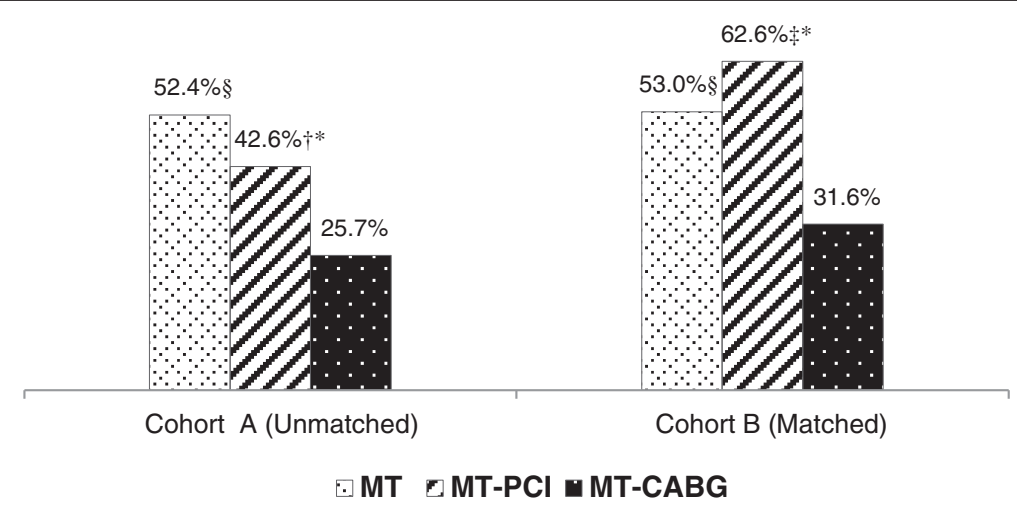

Figure 2 Smoker who kept smoking at one year after angiography. MT: Medial Treatment, PCl: Percutaneous Coronary Intervention, CABG: Coronary Artery Bypass Grafting. $\dagger p$ value PCI vs. MT $<0.001 ;{ }^{*} p$ value $P C I$ vs. CABG $<0.001$; $\$ p$ value $M T$ vs. CABG $<0.001 ; \neq p$ value $\mathrm{PCl}$ vs. $\mathrm{MT}=0.007$. 
Table 1 Baseline characteristics stratified by smoking status at angiography (Cohort $A$, unmatched)

\begin{tabular}{|c|c|c|c|}
\hline Clinical characteristics & Non-smoker & Smoker & p-value \\
\hline No. (\%) & $8750(77.2)$ & $2584(22.8)$ & \\
\hline Age, Mean (SD) & $66.4(10.4)$ & $58.7(9.8)$ & $<0.001$ \\
\hline Female (\%) & 21.8 & 22.4 & \\
\hline Diabetes Mellitus (\%) & 22.9 & 19.6 & $<0.001$ \\
\hline Hyperlipidemia (\%) & 78.5 & 79.2 & \\
\hline Pulmonary disease (\%) & 12.6 & 14.0 & \\
\hline Heart Failure (\%) & 8.7 & 9.1 & \\
\hline Hypertension (\%) & 70.5 & 62.6 & $<0.001$ \\
\hline CVD (\%) & 5.9 & 4.1 & $<0.001$ \\
\hline PVD (\%) & 5.9 & 7.2 & 0.015 \\
\hline ST-Elevated MI (\%) & 21.5 & 38.2 & $<0.001$ \\
\hline Non ST-Elevated MI (\%) & 21.9 & 29.3 & $<0.001$ \\
\hline Prior MI (\%) & 22.4 & 24.9 & 0.008 \\
\hline Prior PCI (\%) & 4.8 & 3.3 & 0.001 \\
\hline Prior CABG (\%) & 3.7 & 1.4 & $<0.001$ \\
\hline Prior lytic (\%) & 4.8 & 10.6 & $<0.001$ \\
\hline ACS (\%) & 55.5 & 73.8 & $<0.001$ \\
\hline Indication for angiography (\%) & & & $<0.001$ \\
\hline Stable angina & 37.7 & 20.7 & \\
\hline $\mathrm{Ml}$ & 38.9 & 60.0 & \\
\hline Unstable angina & 18.6 & 15.1 & \\
\hline Other* & 4.9 & 4.1 & \\
\hline Priority to angiography (\%) & & & $<0.001$ \\
\hline Emergency & 10.5 & 16.1 & \\
\hline Urgent in Hospital & 47.2 & 59.0 & \\
\hline Urgent out of hospital & 14.5 & 9.2 & \\
\hline Planned & 27.1 & 14.4 & \\
\hline Unknown & 0.8 & 1.3 & \\
\hline Ejection fraction (\%) & & & $<0.001$ \\
\hline$>50 \%$ & 62.3 & 57.5 & \\
\hline $35-50 \%$ & 17.6 & 22.1 & \\
\hline $20-34 \%$ & 3.6 & 4.6 & \\
\hline$<20 \%$ & 0.8 & 1.2 & \\
\hline Not done & 8.7 & 7.8 & \\
\hline Coronary Anatomy (\%) & & & $<0.001$ \\
\hline Normal & 0 & 0 & \\
\hline$<50 \%$ disease & 0.2 & 0.1 & \\
\hline Low risk (1-2 VD) & 53.2 & 61.3 & \\
\hline High Risk (2-3 VD) & 36.7 & 30.9 & \\
\hline Left Main disease & 9.8 & 7.6 & \\
\hline
\end{tabular}

Table 1 Baseline characteristics stratified by smoking status at angiography (Cohort $\boldsymbol{A}$, unmatched) (Continued)

\begin{tabular}{llll}
\hline First treatment after angiography (\%) & & & $<0.001$ \\
Medical & 21.4 & 17.6 & \\
CABG & 22.8 & 18.4 & \\
PCl & 55.8 & 64.0 & \\
\hline
\end{tabular}

SD: Standard deviation, BMI: body mass index, CVD: Cerebrovascular disease, PVD: Peripheral vascular disease, MI: myocardial infarction, PCl: Percutaneous Coronary Intervention, CABG: Coronary Artery Bypass Grafting, ACS: Acute coronary syndrome, VD: vessel disease. *Other include silence ischemia, evaluation of serious arrhythmia, preoperative assessment, research protocol angiography.

of Myocardial Infarction 2 study [13]. We found that smokers were more likely to present on an urgent basis and suffer an STEMI relative to non-smokers. Despite successful efforts to promote a standardized approach to the management of patients following acute myocardial infarction (AMI), the prevalence of smoking in this population group has remained largely unchanged over the last decade [14].

A key treatment goal following the diagnosis of CAD is to initiate effective secondary prevention measures, including nicotine addiction management for those patients who smoke [10]. Our data point to the importance of rehabilitation care for smokers following diagnostic or any procedural interventions, particularly those who undergo PCI. In Alberta, patients who have been diagnosed with CAD through angiography are referred to outpatient cardiac risk management programs. Unfortunately, data on referral rates by clinicians and uptake of these programs by patients were unavailable, nevertheless, evidence from other sources indicate that referral to out-patient cardiac rehabilitation services remains low [15] especially in the socially disadvantaged [16]. Recent Medicare data indicate that those who have undergone CABG are more than twice as likely as others to use out-patient cardiac rehabilitation services [16]. Parashar et al. [17] tracked participation rates of patients after an AMI enrolled into cardiac rehabilitation programs after discharge from hospital and found that current smokers, those with lower socioeconomic status and those who had undergone PCI were the least likely to participate in the programs.

To our knowledge, this is the largest study to assess the impact of smoking and smoking cessation on outcomes in a broad and generalizable propensity matched cohort. We found a strong association with a lower survival in patients who smoked who had undergone revascularization, even after adjusting for key variables and performing a propensity matching to account for variations in patient and clinician decisions on further care.

For smokers with CAD, quitting smoking is the most effective secondary preventive measure in reducing mortality 
Table 2 Baseline characteristics stratified by treatment (Cohort A)

\begin{tabular}{|c|c|c|c|}
\hline Clinical characteristics & Medical & Revascularization & $p$-value \\
\hline No. (\%) & $2326(20.5)$ & $9008(79.5)$ & \\
\hline BMI, Mean (SD) & $28.8(5.6)$ & $28.8(5.1)$ & \\
\hline Age, Mean (SD) & $65.5(10.8)$ & $64.2(10.7)$ & $<0.001$ \\
\hline Female (\%) & 24.9 & 21.1 & $<0.001$ \\
\hline Current smoker (\%) & 19.6 & 23.6 & $<0.001$ \\
\hline Diabetes Mellitus (\%) & 26.9 & 20.9 & $<0.001$ \\
\hline Hyperlipidemia (\%) & 78.9 & 78.6 & \\
\hline Pulmonary disease (\%) & 16.3 & 12.1 & $<0.001$ \\
\hline Heart Failure (\%) & 13.7 & 7.5 & $<0.001$ \\
\hline Hypertension (\%) & 72.8 & 67.6 & $<0.001$ \\
\hline CVD (\%) & 7.5 & 5.0 & $<0.001$ \\
\hline PVD (\%) & 8.5 & 5.6 & $<0.001$ \\
\hline ST-Elevated MI (\%) & 11.7 & 28.9 & $<0.001$ \\
\hline Non ST-Elevated MI (\%) & 19.1 & 24.8 & $<0.001$ \\
\hline Prior MI (\%) & 28.3 & 21.6 & $<0.001$ \\
\hline Prior PCI (\%) & 5.7 & 4.1 & 0.001 \\
\hline Prior CABG (\%) & 7.2 & 2.1 & $<0.001$ \\
\hline Prior lytic (\%) & 2.6 & 7.0 & $<0.001$ \\
\hline ACS (\%) & 42.8 & 64.1 & $<0.001$ \\
\hline Indication for angiography (\%) & & & $<0.001$ \\
\hline Acute/recent MI & 26.7 & 48.1 & \\
\hline Unstable angina & 17.4 & 17.9 & \\
\hline Stable angina & 48.1 & 30.1 & \\
\hline Other* & 7.8 & 3.9 & \\
\hline Priority to angiography (\%) & & & $<0.001$ \\
\hline Emergency & 2.2 & 14.2 & \\
\hline Urgent in hospital & 45.5 & 51.0 & \\
\hline Urgent out of hospital & 16.8 & 12.3 & \\
\hline Planned & 35.4 & 21.4 & \\
\hline Unknown & 0.2 & 1.0 & \\
\hline Ejection fraction (\%) & & & $<0.001$ \\
\hline$>50 \%$ & 62.0 & 61.0 & \\
\hline $35-50 \%$ & 16.0 & 19.3 & \\
\hline $20-34 \%$ & 6.0 & 3.2 & \\
\hline$<20 \%$ & 2.0 & 0.6 & \\
\hline Not done & 13.9 & 15.9 & \\
\hline Coronary Anatomy (\%) & & & $<0.001$ \\
\hline Normal & 0.2 & 0.0 & \\
\hline$<50 \%$ disease & 0.3 & 0.1 & \\
\hline Low risk (1-2 VD) & 65.5 & 52.4 & \\
\hline High Risk (2-3 VD) & 28.6 & 37.1 & \\
\hline Left Main disease & 5.4 & 10.3 & \\
\hline
\end{tabular}

SD: Standard deviation, BMI: body mass index, CVD: Cerebrovascular disease, PVD: Peripheral vascular disease, Ml: myocardial infarction, PCI: Percutaneous Coronary Intervention, CABG: Coronary Artery Bypass Grafting, ACS: Acute coronary syndrome, VD: vessel disease. *Other include silence ischemia, evaluation of serious arrhythmia, preoperative assessment, research protocol angiography.
Table 3 Baseline characteristics by smoking status at one year after angiography (Cohort $A$ )

\begin{tabular}{lccl}
\hline Clinical characteristics & $\begin{array}{c}\text { Smoker at } \\
\text { one year }\end{array}$ & $\begin{array}{c}\text { Quitter at } \\
\text { one year }\end{array}$ & p-value \\
\hline No. (\%) & $1064(41.2)$ & $1519(58.8)$ & \\
BMI, Mean (SD) & $28.6(5.6)$ & $28.8(5.2)$ & 0.4 \\
Age, Mean (SD) & $58.4(9.6)$ & $58.9(9.9)$ & 0.3 \\
Female (\%) & 27.3 & 19.0 & $<0.001$ \\
Diabetes Mellitus (\%) & 19.0 & 20.1 & 0.5 \\
Hyperlipidemia (\%) & 79.7 & 78.9 & 0.6 \\
Pulmonary disease (\%) & 15.3 & 13.1 & 0.1 \\
Heart Failure (\%) & 8.4 & 9.6 & 0.3 \\
Hypertension (\%) & 63.1 & 62.3 & 0.7 \\
CVD (\%) & 4.4 & 3.9 & 0.6 \\
PVD (\%) & 8.9 & 6.1 & 0.007 \\
ST-Elevated MI (\%) & 34.7 & 40.6 & 0.002 \\
Non ST-Elevated Ml (\%) & 27.3 & 30.7 & 0.07 \\
Prior MI (\%) & 26.2 & 23.9 & 0.2 \\
Prior PCl (\%) & 3.8 & 3.0 & 0.3 \\
Prior CABG (\%) & 1.3 & 1.4 & 0.9 \\
Prior lytic (\%) & 8.6 & 12.0 & 0.006 \\
ACS (\%) & 70.2 & 76.4 & 0.001 \\
Indication for angiography (\%) & & & $<0.001$
\end{tabular}

$\begin{array}{lcc}\text { Acute/recent MI } & 55.0 & 63.5 \\ \text { Unstable angina } & 16.1 & 14.5 \\ \text { Stable angina } & 24.9 & 17.8 \\ \text { Other* } & 4.0 & 4.2\end{array}$

Priority to angiography (\%)

$\begin{array}{lcc}\text { Emergency } & 13.8 & 17.7 \\ \text { Urgent in hospital } & 58.2 & 59.5 \\ \text { Urgent out of hospital } & 10.1 & 8.6 \\ \text { Planned } & 16.5 & 13.0 \\ \text { Unknown } & 1.4 & 1.2\end{array}$

Ejection fraction (\%)

$\begin{array}{lcc}>50 \% & 59.8 & 56.0 \\ 35-50 \% & 22.3 & 21.9 \\ 20-34 \% & 4.1 & 4.8 \\ <20 \% & 0.9 & 1.4 \\ \text { Not done } & 14.9 & 16.0\end{array}$

Coronary Anatomy (\%)

$\begin{array}{lcc}\text { Normal } & 0.0 & 0.1 \\ <50 \% \text { disease } & 0.0 & 0.2 \\ \text { Low risk (1-2 VD) } & 69.9 & 55.2 \\ \text { High Risk (2-3 VD) } & 25.1 & 35.0 \\ \text { Left Main disease } & 4.9 & 9.5\end{array}$

SD: Standard deviation, BMI: body mass index, CVD: Cerebrovascular disease, PVD: Peripheral vascular disease, MI: myocardial infarction, PCl: Percutaneous Coronary Intervention, CABG: Coronary Artery Bypass Grafting, ACS: Acute coronary syndrome, VD: vessel disease. *Other include silence ischemia, evaluation of serious arrhythmia, preoperative assessment, research protocol angiography. 
Table 4 Baseline characteristics stratified by treatment (Cohort B)

\begin{tabular}{|c|c|c|c|}
\hline Clinical characteristics & Medical & Revascularization & p-value \\
\hline No. & 2123 & 2123 & \\
\hline BMI, Mean (SD) & $28.8(5.6)$ & $28.8(5.2)$ & 1 \\
\hline Age, Mean (SD) & $66.5(10.8)$ & $66(10.3)$ & 0.2 \\
\hline Female (\%) & 24.6 & 25.4 & 0.6 \\
\hline Current smoker (\%) & 18.6 & 18.5 & 1 \\
\hline Renal disease (\%) & 3.9 & 3.9 & 1 \\
\hline Liver/ Gl disease (\%) & 8.2 & 8.9 & 0.4 \\
\hline Diabetes Mellitus (\%) & 26.6 & 25.6 & 0.4 \\
\hline Hyperlipidemia (\%) & 78.8 & 78.9 & 1 \\
\hline Malignancies (\%) & 5.1 & 5.3 & 0.7 \\
\hline Pulmonary disease (\%) & 16.9 & 17.2 & 0.8 \\
\hline Heart Failure (\%) & 13.8 & 12.0 & 0.07 \\
\hline Hypertension (\%) & 72.4 & 72.5 & 1 \\
\hline CVD (\%) & 7.6 & 8.0 & 0.7 \\
\hline PVD (\%) & 8.6 & 8.7 & 0.9 \\
\hline ST-Elevated MI (\%) & 11.4 & 10.7 & 0.5 \\
\hline Non ST-Elevated MI (\%) & 18.8 & 18.6 & 0.8 \\
\hline Prior MI (\%) & 28.0 & 27.5 & 0.7 \\
\hline Prior PCI (\%) & 5.9 & 6.2 & 0.6 \\
\hline Prior CABG (\%) & 7.2 & 5.9 & 0.1 \\
\hline Prior lytic (\%) & 2.7 & 2.7 & 1 \\
\hline ACS (\%) & 42.1 & 41.9 & 0.9 \\
\hline Indication for angiography (\%) & & & 0.9 \\
\hline Acute/recent Ml & 26.1 & 25.5 & \\
\hline Unstable angina & 17.1 & 17.6 & \\
\hline Stable angina & 49.3 & 49.0 & \\
\hline Other* & 7.5 & 7.9 & \\
\hline Ejection fraction (\%) & & & 0.1 \\
\hline$>50 \%$ & 63.0 & 66.0 & \\
\hline $35-50 \%$ & 16.0 & 14.6 & \\
\hline $20-34 \%$ & 6.2 & 5.1 & \\
\hline$<20 \%$ & 1.8 & 1.3 & \\
\hline Not done & 7.0 & 6.2 & \\
\hline Coronary Anatomy (\%) & & & 0.7 \\
\hline Normal & 0.2 & 0 & \\
\hline$<50 \%$ disease & 0.3 & 0.3 & \\
\hline Low risk (1-2 VD) & 66.1 & 67.2 & \\
\hline High Risk (2-3 VD) & 28.3 & 27.5 & \\
\hline Left Main disease & 5.1 & 4.9 & \\
\hline
\end{tabular}

SD: Standard deviation, BMI: body mass index, CVD: Cerebrovascular disease, PVD: Peripheral vascular disease, MI: myocardial infarction, PCI: Percutaneous Coronary Intervention, CABG: Coronary Artery Bypass Grafting, ACS: Acute coronary syndrome, VD: vessel disease. *Other include silence ischemia, evaluation of serious arrhythmia, preoperative assessment, research protocol angiography.
Table 5 Treatments received after first treatment during follow-up period (Cohort B)

\begin{tabular}{|c|c|c|c|c|c|c|c|}
\hline & & \multicolumn{6}{|c|}{ First treatment after catheterization } \\
\hline & & \multicolumn{2}{|c|}{ Medical } & \multicolumn{2}{|c|}{ CABG } & \multicolumn{2}{|c|}{$\mathrm{PCl}$} \\
\hline & & $\mathbf{N}$ & $\%$ & $\mathbf{N}$ & $\%$ & $\mathbf{N}$ & $\%$ \\
\hline \multirow{8}{*}{$\begin{array}{l}\text { Secondary } \\
\text { treatment }\end{array}$} & None & 1818 & 85.6 & 487 & 87.1 & 1359 & 86.9 \\
\hline & $\mathrm{PCl}$ & 95 & 4.5 & 39 & 7.0 & 59 & 3.8 \\
\hline & CABG & 124 & 5.8 & 21 & 3.8 & 73 & 4.7 \\
\hline & Lytic & 56 & 2.6 & 3 & 0.5 & 47 & 3.0 \\
\hline & PCl \& Lytic & 1 & 0.0 & 0 & 0.0 & 3 & 0.2 \\
\hline & CABG \& Lytic & 0 & 0.0 & 0 & 0.0 & 1 & 0.1 \\
\hline & $P C l \& C A B G$ & 28 & 1.3 & 7 & 1.3 & 21 & 1.3 \\
\hline & PCI \& CABG \& Lytic & 1 & 0 & 2 & 0.4 & 1 & 0.1 \\
\hline Total & & 2123 & 100.0 & 559 & 100.0 & 1564 & 100.0 \\
\hline
\end{tabular}

$[18,19]$. The evidence that quitting smoking is associated with a survival benefit relative to continuing to smoke is overwhelming. These data, first elegantly described in a prospective cohort study by Richard Doll in the British Doctors Study [20], have been recently re-enforced by the Nurses' Health Study conducted in over 100,000 women between 1982 and 2004 [21]. In a systematic review of twenty observational non-randomized studies, Critchley et al. [22] found that quitting smoking was associated with substantial risk reduction (36\%) of all-cause mortality among patients with CAD. The risk reduction for other secondary prevention measures was $29 \%$ for statins [23], 15\% for Aspirin [24], 23\% for $\beta$-blockers [25], and $23 \%$ for angiotensin converting enzyme inhibitors [26]. An earlier systematic review found comparable risk reduction [27].

Among patients with CAD, quitting smoking reduces the risk of recurrent events, improves patients' quality of life and is cost-effective [28,29]. Compared with other secondary preventive measures, smoking cessation is both an effective and cost-effective intervention [18,22]. Our data provide an impetus to health systems, policy makers and patients to ensure this remains a focus of care regardless of the CAD management strategy. The Canadian Cardiovascular Society has emphasised the priority of identifying and documenting smoking status and providing smoking cessation support for all smokers admitted to hospital with CAD [10]. The outcome of implementing such programs highly depends on the program structure and duration. Short inpatient education programs have not increased smoking cessation rates at one year and at five years [30]. More intensive behavioral interventions with at least one month of supportive contacts after hospital discharge increased cessation rates [31,32], as does add pharmacological management $[30,33]$. 
Table 6 Treatment association with self-reported smoking status at 1 year post angiography

\begin{tabular}{lccccccc}
\hline Smoking status at & \multicolumn{3}{c}{ Treatment } & & \multicolumn{3}{c}{$\mathbf{X}^{\mathbf{2}}$ (p-value) } \\
\cline { 2 - 4 } & MT no. (\%) & CABG no. (\%) & PCI no. (\%) & & MT vs. CABG & MT vs. PCI & CABG vs. PCI \\
\hline Cohort A smokers quit at one year & $217(47.6)$ & $353(74.3)$ & $949(57.4)$ & & $69.9(<0.001)$ & $14.0(<0.001)$ & $44.2(<0.001)$ \\
Cohort B smokers quit at one year & $185(47.0)$ & $65(68.4)$ & $111(37.4)$ & & $14.11(<0.001)$ & $6.35(0.007)$ & $28.05(<0.001)$ \\
\hline
\end{tabular}

PCI: Percutaneous Coronary Intervention, CABG: Coronary Artery Bypass Grafting.

We observed lower mortality rates across intervention groups when data were analysed into two successive 45 -month periods. These findings could be a reflection of an improvement in CAD management strategies. Despite this, we found long-term survival rates were consistently lower among patients with CAD who smoked compared to non-smokers or smokers who quit smoking. The observed reduction in mortality is similar to the results reported in the Coronary Artery Surgery Study after 10 years of follow-up [34], where patients who continued to smoke had significantly lower survival rates compared with quitters in CABG group $(84 \%$ vs. $68 \%, p=0.018)$ and not a significant difference for patients in the medical treatment arm (75\% vs. $71 \%)$.

Observations that smokers had decreased mortality rates following acute myocardial infarction, in the era when fibrinolysis was the leading reperfusion strategy, have introduced the term "smokers' paradox" to the medical literature. However, our findings, as well other recent studies do not support this observation [35]. Likewise, a recent systematic review reported that all contemporary studies that follow-up with patients for longer than one

Table 7 Survival analysis

\begin{tabular}{|c|c|c|c|c|c|}
\hline \multicolumn{6}{|c|}{ Cohort $A$ (Unmatched) } \\
\hline & \multirow[t]{2}{*}{ Smoking status } & \multirow[t]{2}{*}{ Total N } & \multirow{2}{*}{$\begin{array}{c}\mathrm{N} \text { of } \\
\text { events }\end{array}$} & \multicolumn{2}{|c|}{ Censored } \\
\hline & & & & $\mathbf{N}$ & Percent \\
\hline & Smokers & 1064 & 85 & 979 & $92.0 \%$ \\
\hline & $\begin{array}{l}\text { Smokers who quit } \\
\text { at one year }\end{array}$ & 1519 & 65 & 1454 & $95.7 \%$ \\
\hline & Non-smokers & 8606 & 610 & 7996 & $92.9 \%$ \\
\hline \multicolumn{6}{|c|}{ Cohort B (matched) } \\
\hline \multirow[t]{2}{*}{ Treatment } & \multirow[t]{2}{*}{ Smoking status } & \multirow[t]{2}{*}{ Total $\mathbf{N}$} & \multirow{2}{*}{$\begin{array}{c}\mathrm{N} \text { of } \\
\text { events }\end{array}$} & \multicolumn{2}{|c|}{ Censored } \\
\hline & & & & $\mathrm{N}$ & Percent \\
\hline \multirow{4}{*}{ MT } & Smokers & 209 & 25 & 184 & $88.0 \%$ \\
\hline & $\begin{array}{l}\text { Smokers who quit } \\
\text { at one year }\end{array}$ & 185 & 13 & 172 & $93.0 \%$ \\
\hline & Non-smokers & 1697 & 184 & 1513 & $89.2 \%$ \\
\hline & Smokers ${ }^{* \S}$ & 216 & 24 & 192 & $88.9 \%$ \\
\hline \multirow[t]{2}{*}{ RV } & $\begin{array}{l}\text { Smokers who quit } \\
\text { at one year }\end{array}$ & 176 & 9 & 167 & $94.9 \%$ \\
\hline & Non-smokers & 1699 & 123 & 1576 & $92.8 \%$ \\
\hline
\end{tabular}

*Log rank significance compared to smokers who quit at one year $<0.05$.

${ }^{\S}$ Log rank significance compared to non-smokers $<0.05$.

MT: Medical Treatment; RV: Revascularization. year do not have evidence for such paradox attributing the existence of this phenomenon to the difference in the baseline risk and comorbidities, to the difference in the pathogenesis of acute coronary events between smokers and non-smokers, or to methodological errors in studies [36]. Our data lend further evidence that quitting smoking, particularly if revascularization has been performed, is beneficial.

We have shown in our study, albeit in a relatively small sample size, a trend of greater long term survival rate for smokers who self-reported quitting at one year compared with non-smokers. The mechanisms underlying this effect were not the focus of this study. However, the deleterious consequences of smoking on stress hormone release, blood pressure, thrombotic tendency and vascular biology have been well described and aptly summarized in a recent review by Messner and Bernard [37].

\section{Strengths and limitations}

This study used data from an observational registry and has limitations that generally apply to observational analyses. The APPROACH registry captures all patients for the catchment area in Alberta (population 3.5 million) and has complete data on all patients undergoing angiography in the province and their subsequent treatment and outcome. Hence, it is highly generalizable and reflects clinical care but may not be able to capture all variables that influence survival or would eliminate or enhance any of our statistically significant results. Variables usually absent from larger observational datasets including number of diseased coronary arteries, EF and coronary anatomy are available enhancing the ability to better describe the population being studied. Propensitybased matching attempts to eliminate other bias associated with conventional multivariable modeling and treatment selection. This study used self-reported smoking cessation rates to assess smoking status and survey methodology. Although biochemical validation of smoking status is considered a more robust method in assessing cessation, self-reported cessation rates are still a reliable method in assessing smoking status in patients admitted to hospital [38]. Unfortunately, we do not know the duration of smoking history or life time exposure to tobacco of our population; these data were not included in the Approach database. 


\section{Conclusions}

Our data indicate that smokers who have undergone CABG were more likely to report smoking cessation at one year following the angiography than those who are treated with PCI or MT. Quitting smoking is associated with improved long-term survival, particularly among revascularized patients. Smoking remains an undertreated risk factor among patients with angiographically proven CAD. Quitting smoking should be a key objective of management in patients with CAD.

\section{Competing interests}

The authors declare that they have no competing interests. JAE receives salary support as a Population Health Investigator from Alberta Innovates Health Solutions.

\section{Authors' contributions}

All authors have participated in the work and have reviewed and agree with the content of the article. FH was responsible for the study concept and design, statistical analysis and interpretation of data, drafting of the manuscript and critical revision of the manuscript for important intellectual content. JAE was responsible for the study concept and design, analysis and interpretation of data, drafting the manuscript and critical revision of the manuscript for important intellectual content. CN was responsible for study concept and design, statistical analysis and interpretation of data, drafting of the manuscript and critical revision of the manuscript for important intellectual content. CTW was responsible for study concept and design and critical revision of the manuscript for important intellectual content. BF was responsible for the study concept and design, interpretation of data and critical revision of the manuscript for important intellectual content. All authors read and approved the final manuscript.

\section{Acknowledgements}

We would like to acknowledge Alyssa Chappell and Katherine Pohoreski for revising the article in preparation for publication. This study was funded internally by the Department of Anesthesiology and Pain Medicine, University of Alberta.

\section{Author details}

${ }^{1}$ Department of Anesthesiology \& Pain Medicine, University of Alberta, 8-120 Clinical Sciences Building, Edmonton, Alberta T6G2G3, Canada. ${ }^{2}$ Mazankowsk Alberta Heart Institute, Edmonton, Alberta, Canada. ${ }^{3}$ Faculty of Nursing, University of Alberta, Edmonton, Alberta, Canada. ${ }^{4}$ School of Public Health, University of Alberta, Edmonton, Alberta, Canada. ${ }^{5}$ Division of Cardiovascular Surgery, University of Alberta, Edmonton, Alberta, Canada. ${ }^{6}$ Division of Cardiology, University of Alberta, Edmonton, Alberta, Canada.

Received: 22 July 2014 Accepted: 22 September 2014 Published: 1 October 2014

\section{References}

1. A Report of the Surgeon General: How tobacco smoke causes disease: the biology and behavioral basis for smoking-attributable disease. 2010, Available at: http://www.surgeongeneral.gov/library/reports/tobaccosmoke/ index.html. Accessed: 2013-09-04. (Archived by WebCite ${ }^{\circledast}$ at http://www. webcitation.org/6J07rC7Gh).

2. van Domburg RT, op Reimer WS, Hoeks SE, Kappetein AP, Bogers AJ: Three life-years gained from smoking cessation after coronary artery bypass surgery: a 30-year follow-up study. Am Heart J 2008, 156:473-476.

3. Ashby DT, Dangas G, Mehran R, Lansky AJ, Fahy MP, lakovou I, Satler LF, Pichard AD, Kent KM, Stone GW, Leon MB: Comparison of one-year outcomes after percutaneous coronary intervention among current smokers, ex-smokers, and nonsmokers. Am J Cardiol 2002, 89:221-224.

4. Sherif MA, Nienaber CA, Toelg R, Abdel-Wahab M, Geist V, Schneider S, Senges J, Kuck KH, Tebbe U, Richardt G: Impact of smoking on the outcome of patients treated with drug-eluting stents: 1-year results from the prospective multicentre German Drug-Eluting Stent Registry (DES.DE). Clin Res Cardiol 2011, 100(5):413-423.
5. Kenfield SA, Stampfer MJ, Rosner BA, Colditz GA: Smoking and smoking cessation in relation to mortality in women. JAMA 2008, 299:2037-2047. doi:10.1001/jama.299.17.2037.

6. Gellert C, Schöttker B, Brenner H: Smoking and all-cause mortality in older people: systematic review and meta-analysis. Arch Intern Med 2012, 172(11):837-844. doi:10.1001/archinternmed.2012.1397.

7. van Domburg RT, Meeter K, van Berkel DF, Veldkamp RF, van Herwerden LA, Bogers AJ: Smoking cessation reduces mortality after coronary artery bypass surgery: a 20-year follow-up study. J Am Coll Cardiol 2000, 36:878-883.

8. Canto JG, Kiefe Cl, Rogers WJ, Peterson ED, Frederick PD, French WJ, Gibson CM, Pollack CV Jr, Ornato JP, Zalenski RJ, Penney J, Tiefenbrunn AJ, Greenland P, NRMI Investigators: Number of coronary heart disease risk factors and mortality in patients with first myocardial infarction. JAMA 2011, 306:2120-2127. doi:10.1001/jama.2011.1654.

9. Centers for Disease Control and Prevention (CDC): Smoking attributable mortality, years of potential life lost, and productivity losses - United States, 2000-2004. 2008, 57(45):p.1226. Available at: http://www.cdc.gov/ mmwr/PDF/wk/mm5745.pdf. Accessed: 2013-09-04. (Archived by WebCite at http://www.webcitation.org/6JO7fH H2A).

10. Pipe AL, Eisenberg MJ, Gupta A, Reid RD, Suskin NG, Stone JA: Smoking cessation and the cardiovascular specialist: Canadian cardiovascular society position paper. Can J Cardiol 2011, 27:132-137. doi:10.1016/j. cjca.2010.12.060

11. Erhardt L: Cigarette smoking: an undertreated risk factor for cardiovascular disease. Atherosclerosis 2009, 205:23-32. doi:10.1016/j. atherosclerosis.2009.01.007

12. Ghali WA, Knudtson ML: Overview of the alberta provincial project for outcome assessment in coronary heart disease. On behalf of the APPROACH investigators. Can J Cardiol 2000, 16:1225-1230.

13. Gourlay SG, Rundle AC, Barron HV: Smoking and mortality following acute myocardial infarction: results from the national registry of myocardial infarction 2 (NRMI 2). Nicotine Tob Res 2002, 4:101-107.

14. Boyer NM, Laskey WK, Cox M, Hernandez AF, Peterson ED, Bhatt DL, Cannon CP, Fonarow GC: Trends in clinical, demographic, and biochemical characteristics of patients with acute myocardial infarction from 2003 to 2008: a report from the american heart association get with the guidelines coronary artery disease program. J Am Heart Assoc 2012, 1:e001206. doi:10.1161/JAHA.112.001206

15. Arena R, Williams M, Forman DE, Cahalin LP, Coke L, Myers J, Hamm L, Kris-Etherton P, Humphrey R, Bittner V, Lavie CJ: Increasing referral and participation rates to outpatient cardiac rehabilitation: the valuable role of healthcare professionals in the inpatient and home health settings: a science advisory from the american heart association. Circulation 2012, 125:1321-1329. doi:10.1161/CIR.0b013e318246b1e5.

16. Suaya JA, Shepard DS, Normand SL, Ades PA, Prottas J, Stason WB: Use of cardiac rehabilitation by medicare beneficiaries after myocardial infarction or coronary bypass surgery. Circulation 2007, 116:1653-1662.

17. Parashar S, Spertus JA, Tang F, Bishop KL, Vaccarino V, Jackson CF, Boyden TF, Sperling L: Predictors of early and late enrollment in cardiac rehabilitation, among those referred, after acute myocardial infarction. Circulation 2012, 126:1587-1595.

18. Woolf $\mathrm{SH}$ : The need for perspective in evidence-based medicine JAMA 1999, 282:2358-2365.

19. Tonstad S, Andrew Johnston J: Cardiovascular risks associated with smoking: a review for clinicians. Eur J Cardiovasc Prev Rehabil 2006, 13:507-514

20. Doll $R$, Peto $R$, Boreham J, Sutherland I: Mortality in relation to smoking: 50 years' observations on male British doctors. BMJ 2004, 328(7455):1519.

21. Kenfield SA1, Wei EK, Rosner BA, Glynn RJ, Stampfer MJ, Colditz GA: Burden of smoking on cause-specific mortality: application to the Nurses' Health Study. Tob Control 2010, 19(3):248-254. doi:10.1136/tc.2009.032839.

22. Critchley JA, Capewell S: Mortality risk reduction associated with smoking cessation in patients with coronary heart disease: a systematic review. JAMA 2003, 290:86-97.

23. Pignone M, Phillips C, Mulrow C: Use of lipid lowering drugs for primary prevention of coronary heart disease: meta-analysis of randomised trials. BMJ 2000, 321:983-986.

24. Antithrombotic Trialists' Collaboration: Collaborative meta-analysis of randomised trials of antiplatelet therapy for prevention of death, myocardial infarction, and stroke in high risk patients. BMJ 2002, 324:71-86. 
25. Freemantle N, Cleland J, Young P, Mason J, Harrison J: Beta blockade after myocardial infarction: systematic review and meta regression analysis. BMJ 1999, 318:1730-1737.

26. Flather MD, Yusuf S, Kober L, Pfeffer M, Hall A, Murray G, Torp-Pedersen C, Ball S, Pogue J, Moyé L, Braunwald E: Long-term ACE-inhibitor therapy in patients with heart failure or left-ventricular dysfunction: A systematic overview of data from individual patients. ACE-inhibitor myocardial infarction collaborative group. Lancet 2000, 355:1575-1581.

27. van Berkel TF, Boersma H, Roos-Hesselink JW, Erdman RA, Simoons ML: Impact of smoking cessation and smoking interventions in patients with coronary heart disease. Eur Heart J 1999, 20(24):1773-1782.

28. Barth J, Critchley J, Bengel J: Efficacy of psychosocial interventions for smoking cessation in patients with coronary heart disease: a systematic review and meta-analysis. Ann Behav Med 2006, 32(1):10-20.

29. Kahn R, Robertson RM, Smith R, Eddy D: The impact of prevention on reducing the burden of cardiovascular disease. Circulation 2008, 118:576-585. doi:10.1161/CIRCULATIONAHA.108.190186.

30. Rigotti NA, Clair C, Munafo MR, Stead LF: Interventions for smoking cessation in hospitalised patients. Cochrane Database Syst Rev 2012, 5, CD001837. doi:10.1002/14651858.CD001837.pub3.

31. Mohiuddin SM, Mooss AN, Hunter CB, Grollmes TL, Cloutier DA, Hilleman $D E$ : Intensive smoking cessation intervention reduces mortality in high-risk smokers with cardiovascular disease. Chest 2007, 131:446-452.

32. Smith PM, Burgess E: Smoking cessation initiated during hospital stay for patients with coronary artery disease: a randomized controlled trial. CMAJ 2009, 180:1297-1303. doi:10.1503/cmaj.080862.

33. Benowitz NL, Prochaska JJ: Smoking cessation after acute myocardial infarction. J Am Coll Cardiol 2013, 61:533-535. doi:10.1016/j.jacc.2012.11.017.

34. Cavender JB, Rogers WJ, Fisher LD, Gersh BJ, Coggin CJ, Myers WO: Effects of smoking on survival and morbidity in patients randomized to medical or surgical therapy in the coronary artery surgery study (CASS): 10-year follow-up. CASS investigators. J Am Coll Cardiol 1992, 20:287-294.

35. Weisz G, Cox DA, Garcia E, Tcheng JE, Griffin JJ, Guagliumi G, Stuckey TD, Rutherford BD, Mehran R, Aymong E, Lansky A, Grines CL, Stone GW: Impact of smoking status on outcomes of primary coronary intervention for acute myocardial infarction-the smoker's paradox revisited. Am Heart J 2005, 150(2):358-364

36. Aune E, Roislien J, Mathisen M, Thelle DS, Otterstad JE: The "smoker's paradox" in patients with acute coronary syndrome: a systematic review. BMC Med 2011, 9:97. doi:10.1186/1741-7015-9-97.

37. Messner B, Bernhard D: Smoking and cardiovascular disease: mechanisms of endothelial dysfunction and early atherogenesis. Arterioscler Thromb Vasc Biol 2014, 34(3):509-515. doi:10.1161/ATVBAHA.113.300156.

38. Pell JP, Haw SJ, Cobbe SM, Newby DE, Pell AC, Oldroyd KG, Murdoch DL, Pringle SD, Dunn FG, Macintyre PD, Gilbert TJ, Fischbacher CM, Borland W: Validity of self-reported smoking status: comparison of patients admitted to hospital with acute coronary syndrome and the general population. Nicotine Tob Res 2008, 10:861-866. doi:10.1080/14622200802023858.

doi:10.1186/1471-2261-14-133

Cite this article as: Hammal et al: Smoking status and survival: impact on mortality of continuing to smoke one year after the angiographic diagnosis of coronary artery disease, a prospective cohort study. BMC Cardiovascular Disorders 2014 14:133.

\section{Submit your next manuscript to BioMed Central and take full advantage of:}

- Convenient online submission

- Thorough peer review

- No space constraints or color figure charges

- Immediate publication on acceptance

- Inclusion in PubMed, CAS, Scopus and Google Scholar

- Research which is freely available for redistribution

Submit your manuscript at www.biomedcentral.com/submit 